\title{
A.N.Uma *
}

The Music Medicine Unit (MMU) in MGMC\&RI was inaugurated in November, 2010, with the blessings of our Hon'ble Chairman and under the headship of Prof. P.Bharathi. Utmost dedication to patient care is our motto. Since its inception, the MMU has been involved in research along with patient care, and several research publications have been documented in international journals. The MMU focuses on undertaking case studies, which bring an important facet to clinical research-that of personal application. Single cases are special examples drawing attention to anomalies in practice, alerting practitioners to matters of urgent attention.

Music Therapy in India is now fast developing from its rudimentary stage in the 90s. Each and every one of us is subject to the limitations of human perceptions. But the power of music transcends all human perceptions, and in those who are endowed with appropriate musical intelligence, emotional responsiveness and receptivity, it does not fail to elicit miraculous healing effects. Music's emotional effect, which is people's primary motivation for listening, may prepare them to attain or maintain a state of well-being. Be it in any of its many forms, music can evoke powerful emotional reactions in people. Listening to music affects the emotional and cognitive experience of an individual as well as physiological arousal. A couple of articles, illustrated here in the Annals of SBV are on the theme 'Music Therapy Interventions' performed in the Music Medicine Unit, MGMC\&RI. Affect has been equated with emotion, but in the research literature, it is used to refer to expression of emotional states. Affective modification by music has been extensively studied by Sutherland et.al, who have stated that music has the capacity of modifying the negative affect aroused during anxiety- provoking situations, since it stimulates affective schemes that undo or cancel the experience of anxiety. Music also stimulates positive affect which will boost cognitive- congruent schemes, and generation of positive cognitions is effective in reducing anxiety.

The articles in the section of The Harmony also highlight the mechanisms behind sleeping pill addiction, and how music therapy can help in relieving such addictions. Sleeping Pills may be prescribed by physicians for use over a short period of time in the treatment of insomnia. It is however, a known fact that many patients become addicted to sleeping pills. Addiction is not a character defect or a moral deficiency, it's an illness. Cognitive Behavioral Therapy (CBT) is a better, long-term solution for sleeping problems than medications. Apart from CBT, music therapy employing sedative Indian ragas have proved efficacious in reducing the dosage of sleeping pills in those with addiction. At this very moment, in homes, offices, cars, restaurants, and clubs around the world, people are listening to music. Why do people listen to music? Although the answer to this question is undoubtedly complex, we can attempt to shed some light on the issue by examining music preferences. Just as individuals shape their social and physical environments to reinforce their dispositions and self-view, the music they select can serve a similar function.

A couple of articles on biological rhythm and how it impacts the mood have also been given in this Annals of SBV. This is a relatively new field and there is still not an extensive amount of research regarding the importance of biological rhythms. It has not been established whether changes in sleep patterns are a cause of mood disorders, or whether the change in sleep patterns is a result of some other factor such as an anxiety or mood disorder. Most people hear some form of music every day, and music affects people in many ways from emotion regulation to cognitive development, along with providing a means for self-expression.

Music training has been shown to help improve intellectual development and ability, though no connection has been found as to how it affects emotion regulation. In general, the plasticity traits (openness to experience and extraversion) affect music preference more than the stability traits (agreeableness, neuroticism). Gender is another factor that affects music preference. Men tend to use music for more cognitive reasons, while women tend to use music for more emotional reasons. Also, men tend to exclude musical genres from one another more than women do. Active mood is another factor that affects music preference. Aggression has been shown to improve creativity and emotional intensity derived from music. People with aggressive disorders find music to be a powerful emotional outlet.

Younger people tend to place much more significance on music and also use background music more. Nostalgia is the most important feeling that affects music preference here. Music producing nostalgia effects have been shown to 
have large predictive effects on people of all ages. A research study carried out on hypertensives, using Rag Ahir Bhairav is also presented.

The articles in this issue under the section 'THE HARMONY' has been presented as a special issue for the 'Annals of SBV' for wider dissemination and readership.

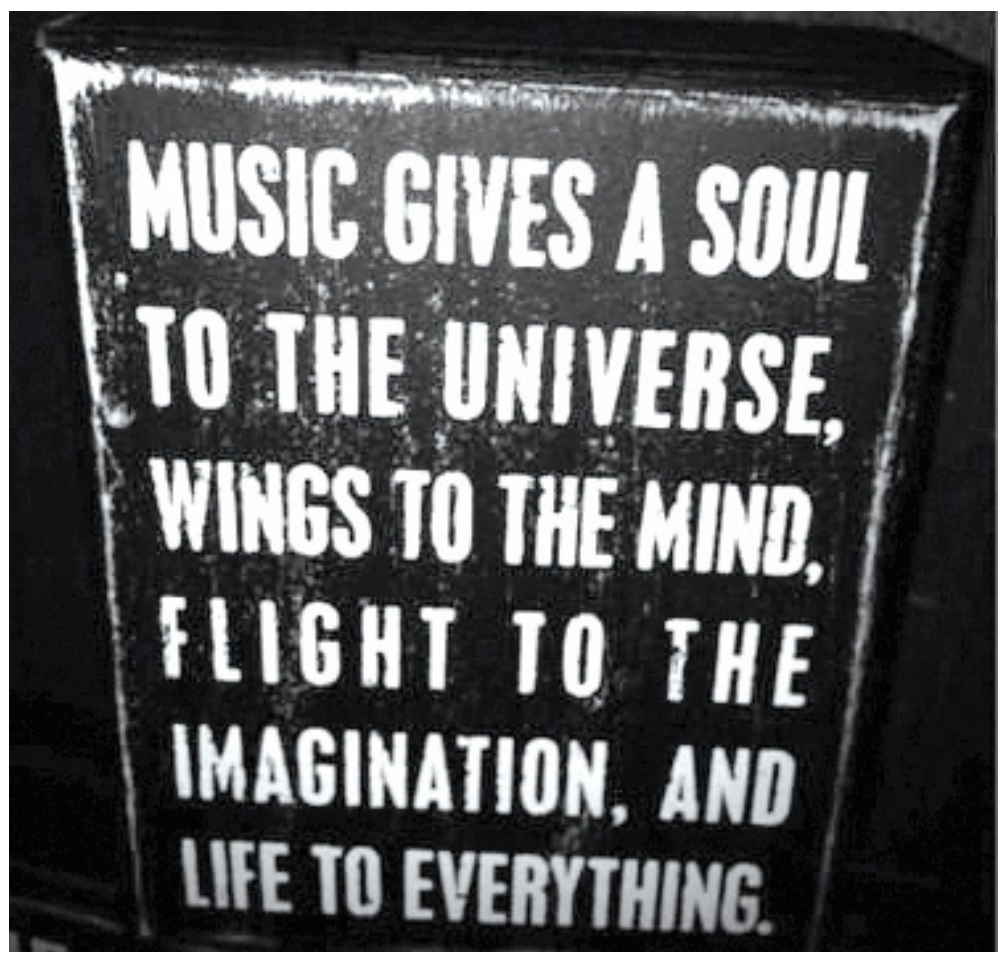

- Plato

"Music is a therapy. It is a communication far more powerful than words, far more immediate, far more efficient."

- Yehudi Menuhin

"The good thing about music is when it hits you, you feel no pain".

W- Bob Marley

* Mrs. A.N.Uma. MSc.,MPhil.,Phd.,MA.,BEd

Asst. Prof., Anatomy Dept.

Executive Editor, THE HARMONY, MGMCRI, Pondicherry 